\title{
Quiste mesentérico gigante: reporte de caso
}

\section{Giant mesenteric cyst. Case report}

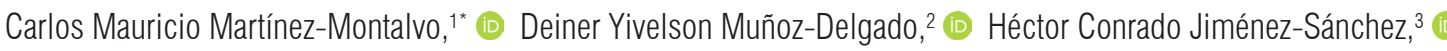

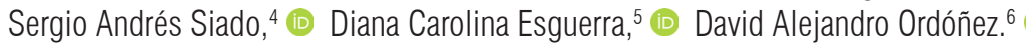

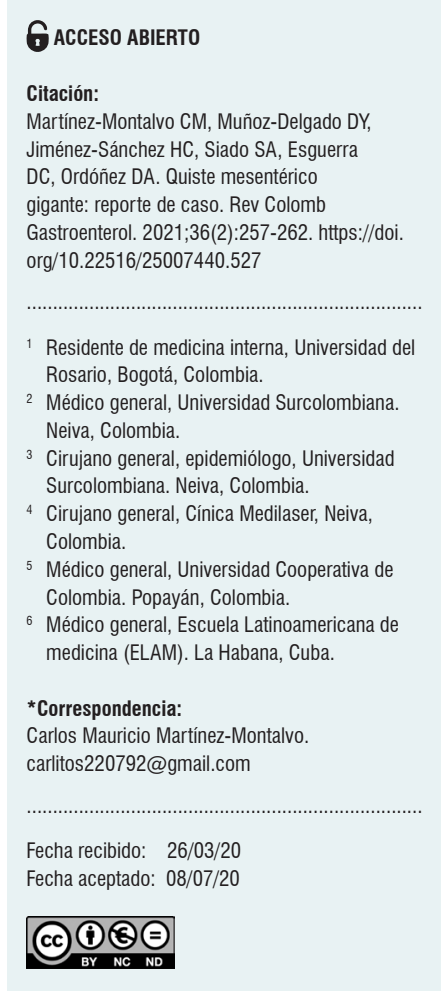

\section{Resumen}

El quiste mesentérico es una patología intraabdominal poco frecuente, en su mayoría benigna. El tratamiento casi siempre es quirúrgico y consiste en la resección del quiste y de los órganos involucrados siempre que sea posible con el fin de reducir la tasa de recurrencia. Se presenta el caso de un paciente de 38 años con dolor abdominal inespecífico y diagnóstico ecográfico de masa retroperitoneal zona II izquierda gigante. Los estudios de extensión incluyeron tomografía axial computarizada, resonancia magnética y endoscopia de vías digestivas altas, cuyos hallazgos informaron una lesión quística gigante. Se realizó resección quirúrgica de la lesión por vía abierta, con diagnóstico histopatológico de quiste mesentérico.

\section{Palabras clave}

Quiste mesentérico, dolor abdominal, radiología, masa abdominal.

\section{Abstract}

A mesenteric cyst is a rare, mostly benign, intra-abdominal tumor. Treatment is almost always surgical and consists of removing the cyst and involved organs whenever possible to prevent recurrence. The following is the case of a 38-year-old patient with nonspecific abdominal pain and an ultrasound diagnosis of a giant retroperitoneal mass in the left medial paracolic gutter. The following imaging studies were performed: computed tomography, magnetic resonance, and endoscopy, finding a giant cystic lesion. An exploratory laparotomy was performed to remove the mass, and a histopathology report confirmed the diagnosis of mesenteric cyst.

\section{Keywords}

Mesenteric cyst; Abdominal pain; Radiology.

\section{INTRODUCCIÓN}

El quiste mesentérico representa una entidad patológica poco frecuente, que puede aparecer en múltiples localizaciones intraabdominales. Fue reportado por primera vez en la autopsia de un niño de 8 años por el anatomista italiano
Benevieni en 1507; posteriormente, en 1800 Tillaux realizó la primera resección quirúrgica de este tipo de lesiones (1). La incidencia actual es de aproximadamente 1 por cada 100000 pacientes adultos y 1 por cada 20000 niños, que acuden a un centro hospitalario (2). La localización más frecuente de estas lesiones es el mesenterio del intestino 
delgado (60\%), seguido del colon ascendente (24\%) y, por último, en la región retroperitoneal (14,5\%) (3). La presentación clínica es diversa: pueden ser cuadros completamente asintomáticos con una posterior aparición de síntomas inespecíficos como dolor abdominal intermitente, distensión abdominal, diarrea o masa abdominal palpable, cuadros de abdomen agudo o de obstrucción intestinal, los cuales se encuentran directamente relacionados con el tamaño de la lesión (4).

Se propuso un sistema de clasificación por Perrot y colaboradores el cual aún se encuentra vigente, en él se clasifica el quiste mesentérico según su origen de la siguiente manera:

1. linfático (simple o linfagioma);

2. mesotelial (simple, quístico benigno o quístico maligno);

3. entérico (quistes de duplicación intestinal o quiste entérico);

4. urogenitales;

5. teratomas; $y$

6. pseudoquistes no pancreáticos (infecciosos o traumáti$\cos )(5)$.

Para su diagnóstico, se utilizan diferentes estudios imagenológicos como la ultrasonografía y tomografía axial computarizada (TAC). Con la primera se evidencian lesiones sólidas, pero no se observan detalles anatómicos; y con la segunda, que es la más utilizada, se puede determinar el contenido del quiste, tamaño de la pared, presencia de tabiques $y$, si se adiciona medio de contraste, se puede obtener información sobre la relación de la masa con estructuras vasculares $\mathrm{u}$ otras estructuras adyacentes $(2,6)$.

Por una parte, el tratamiento de elección es quirúrgico y el abordaje laparoscópico es el preferido, con ventajas sobre otros procedimientos como lograr practicar la enucleación completa del quiste, la recuperación posquirúrgica y la baja tasa de recurrencia. Por otra parte, técnicas como aspiración o marsupialización no son recomendadas debido a las altas tasas de recurrencia y de sobreinfección (7).

En el presente artículo se reporta el caso clínico de un hombre de 38 años sin antecedentes de importancia, que acudió al servicio de urgencias por un cuadro clínico de un día de evolución de dolor abdominal tipo opresivo de intensidad leve, sin criterios de abdomen agudo al ingreso. Por lo anterior, se decidió solicitar una ecografía de abdomen total en la cual se evidenció una masa quística gigante retroperitoneal, se decidió complementar estudios mediante una TAC de abdomen con contraste intravenoso, endosonografía y resonancia magnética de abdomen, con los que se llegó al diagnóstico de quiste mesentérico, por lo cual se decidió llevar a cabo la resección de masa con un abordaje mediante laparotomía debido al tamaño y ubicación de la lesión, con confirmación histopatológica de quiste mesentérico.

\section{OBJETIVO}

Presentar el reporte de caso sobre quiste mesentérico gigante manejado por el servicio de cirugía revisando sus características diagnósticas, manejo y resultado.

\section{MATERIALES}

Un estudio de reporte de caso en el que además se hace una revisión bibliográfica de su enfoque diagnóstico y terapéutico. Se realizó una búsqueda de artículos en bases de datos como Pubmed que incluyera los términos giant mesenteric cyst, haciendo selección de los más relevantes.

\section{RESULTADOS}

Se trata de un paciente masculino de 38 años de edad, residente en Colombia, sin antecedentes personales conocidos, quien consultó por un cuadro clínico de un día de evolución consistente en dolor abdominal, tipo peso de intensidad leve ubicado en el flanco y fosa ilíaca izquierdos sin otra sintomatología asociada. Previo al ingreso, refirió el consumo de analgésicos orales sin presentar mejoría clínica, por lo cual acudió al servicio de urgencias. Al ingreso, en el paciente se registraron signos vitales dentro de los parámetros normales, con un examen abdominal sin presencia de signos de irritación peritoneal, por lo anterior se decidió iniciar el manejo sintomático. Ante la persistencia de la sintomatología se solicitaron paraclínicos, cuyos resultados se presentan en la Tabla 1, los cuales se encontraron dentro de límites normales y, además, se solicitó una ecografía abdominal total, la cual reportó una lesión pasiva por detrás del riñón izquierdo de 160 x 140 × 90 mm, multiseptada, asociada con dilatación pielocalicial izquierda. Ante estos hallazgos se decidió realizar una TAC de abdomen como estudio complementario.

El reporte de la TAC evidenció la presencia de una voluminosa colección que se proyectaba a nivel retroperitoneal izquierdo, que desplazaba el colon y riñón ipsilateral con un contenido heterogéneo y áreas hiperdensas en su interior, formando un nivel, lo que sugirió una colección con diferentes estadios evolutivos de sangrado, en sus mayores diámetros con medidas cerca de 154 x 135 x $100 \mathrm{~mm}$ para un volumen aproximado $180 \mathrm{~mL}$. Las lesiones ejercieron un efecto de masa igualmente en el cuerpo del páncreas y se proyectó hacia la transcavidad de los epiplones. Luego de la administración de contraste, no se observaron áreas de realce por esta lesión; se observó el páncreas de aspecto delgado a 
Tabla 1. Paraclínicos al ingreso

\begin{tabular}{|c|c|c|c|}
\hline \multicolumn{2}{|c|}{ Paraclínicos } & \multirow[b]{2}{*}{$\begin{array}{l}13,9 \mathrm{~g} / \mathrm{dL} \\
42,9 \% \\
10640 \\
82,6 \% \\
10,9 \% \\
188000\end{array}$} & \multirow{2}{*}{$\begin{array}{l}\text { Valores de referencia } \\
12-16 \mathrm{~g} / \mathrm{dL} \\
38 \%-42 \% \\
4500-11000 \\
60 \%-70 \% \\
30 \%-40 \% \\
150000-450000\end{array}$} \\
\hline Hemoleucograma & $\begin{array}{l}\text { Hemoglobina } \\
\text { Hematocrito } \\
\text { Leucocitos } \\
\text { Neutrófilos } \\
\text { Linfocitos } \\
\text { Plaquetas }\end{array}$ & & \\
\hline Citoquímico de orina & $\begin{array}{l}\text { Aspecto } \\
\text { Densidad } \\
\text { Proteínas } \\
\text { Cuerpos cetónicos } \\
\text { Glucosa } \\
\text { Sedimento }\end{array}$ & $\begin{array}{l}\text { Ligeramente turbio } \\
1020 \\
25 \\
\text { Negativo } \\
\text { Negativo } \\
\text { No leucocitos, no eritrocitos, no bacterias }\end{array}$ & $\begin{array}{l}1000-1030 \\
\text { Negativo } \\
\text { Negativo } \\
\text { Negativo }\end{array}$ \\
\hline \multicolumn{2}{|l|}{ Creatinina } & $0,5 \mathrm{mg} / \mathrm{dL}$ & $0,6-1 \mathrm{mg} / \mathrm{dL}$ \\
\hline \multicolumn{2}{|l|}{ Nitrógeno ureico } & $12 \mathrm{mg} / \mathrm{dL}$ & $6-20 \mathrm{mg} / \mathrm{dL}$ \\
\hline \multicolumn{2}{|l|}{ Amilasa } & $34 \mathrm{UI} / \mathrm{mL}$ & $0-137 \mathrm{U} / \mathrm{L}$ \\
\hline \multicolumn{2}{|c|}{ Antígeno carcinoembrionario } & $<0,50 \mathrm{ng} / \mathrm{mL}$ & $<40 \mathrm{ng} / \mathrm{mL}$ \\
\hline \multicolumn{2}{|l|}{ Bilirrubina total } & $1 \mathrm{mg} / \mathrm{dL}$ & $0,1-1,2 \mathrm{mg} / \mathrm{dL}$ \\
\hline \multicolumn{2}{|l|}{ Bilirrubina indirecta } & $1 \mathrm{mg} / \mathrm{dL}$ & $0-0,7 \mathrm{mg} / \mathrm{dL}$ \\
\hline \multicolumn{2}{|l|}{ Fosfatasa alcalina } & $68 \mathrm{U} / \mathrm{L}$ & $50-136 \mathrm{U} / \mathrm{L}$ \\
\hline \multicolumn{2}{|l|}{ Calcio } & $1,187 \mathrm{mmol} / \mathrm{L}$ & $1-2,4 \mathrm{mmol} / \mathrm{L}$ \\
\hline \multicolumn{2}{|l|}{$\mathrm{HbA}_{1 \mathrm{c}}$} & $5,3 \%$ & $<5,6 \%$ \\
\hline \multicolumn{2}{|l|}{ TP } & $10,4 \mathrm{~s}$ & $10-13,5 \mathrm{~s}$ \\
\hline \multicolumn{2}{|l|}{ TTP } & $25,2 \mathrm{~s}$ & $25-35 s$ \\
\hline \multicolumn{2}{|l|}{ INR } & 0,98 & $<1$ \\
\hline \multicolumn{2}{|l|}{ ALT } & $37 \mathrm{U} / \mathrm{L}$ & $30-65 \mathrm{U} / \mathrm{L}$ \\
\hline \multicolumn{2}{|l|}{ AST } & $31 \mathrm{U} / \mathrm{L}$ & $15-37 \mathrm{U} / \mathrm{L}$ \\
\hline \multicolumn{2}{|l|}{ a-fetoproteína } & $1,92 \mathrm{IU} / \mathrm{mL}$ & $<15 \mathrm{IU} / \mathrm{mL}$ \\
\hline \multicolumn{2}{|l|}{ CA 125} & $<4$ & $<35$ \\
\hline
\end{tabular}

ALT: alanina-aminotransferasa; AST: aspartato-aminotransferasa; CA 125: antígeno de cáncer 125; HbA : hemoglobina glicosilada; INR: índice internacional normalizado; TP: tiempo de protrombina; TTP: tiempo de tromboplastina parcial.

nivel del cuerpo y la cola por el efecto compresivo ejercido por la masa previamente descrita, asas intestinales normales, riñones y sistema excretor normal (Figuras 1 y 2 ).

Fue valorado por el servicio de cirugía general, en el que solicitaron marcadores tumorales (Tabla 1) para descartar una lesión neoplásica de páncreas y resonancia magnética para definir el plan quirúrgico, cuyo reporte evidenció una lesión multiseptada del cuerpo y cola del páncreas de $130 \mathrm{x}$ $87 \mathrm{~mm}$ irregular con extensión al bazo, que no tiene plano de clivaje, y estómago fue desplazado hacia la parte superior y anterior (Figura 2); así mismo, se realizó una TAC de alta resolución de tórax como estudio de extensión, la cual fue reportada dentro de los límites normales.

Dados los hallazgos de la resonancia, fue necesario descartar que la lesión fuera dependiente del cuerpo o la cola de páncreas, por lo cual se ordenó la realización de una ultrasonografía endoscópica biliopancreática que reportó una gran lesión multianecoica septada de bordes bien defi- 

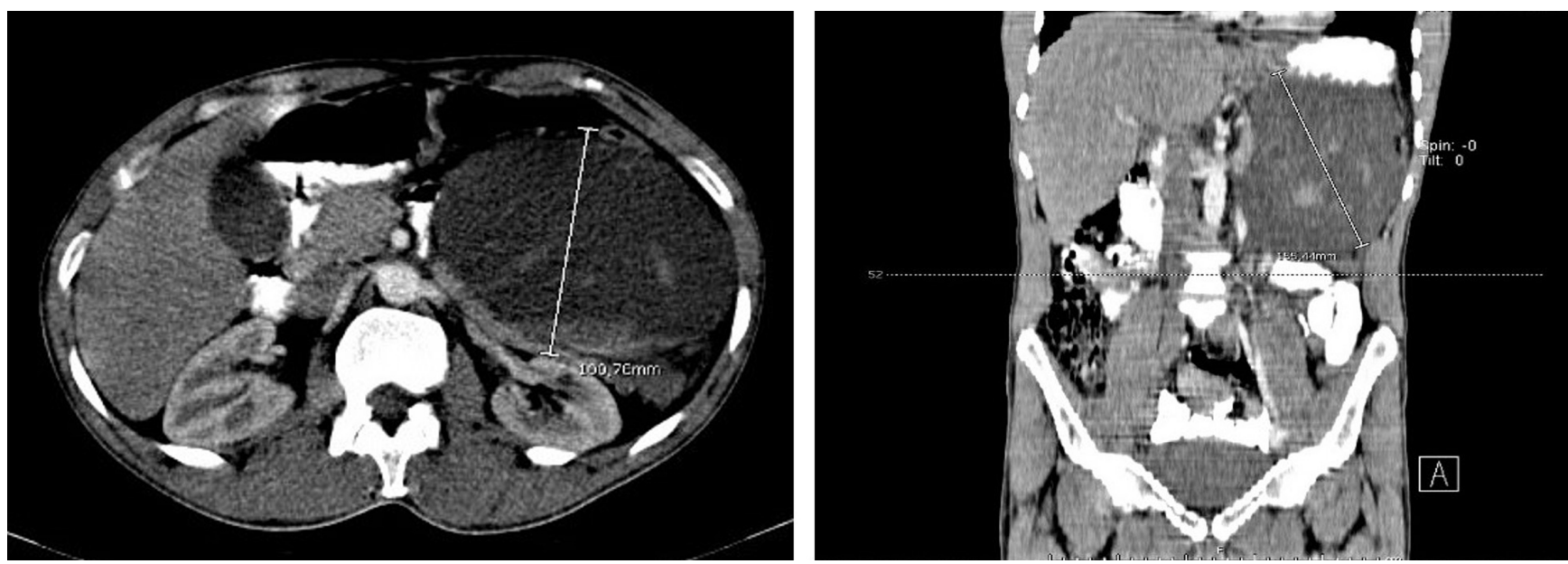

Figura 1. Tomografía de abdomen. A. Fase simple. B. Fase contrastada.
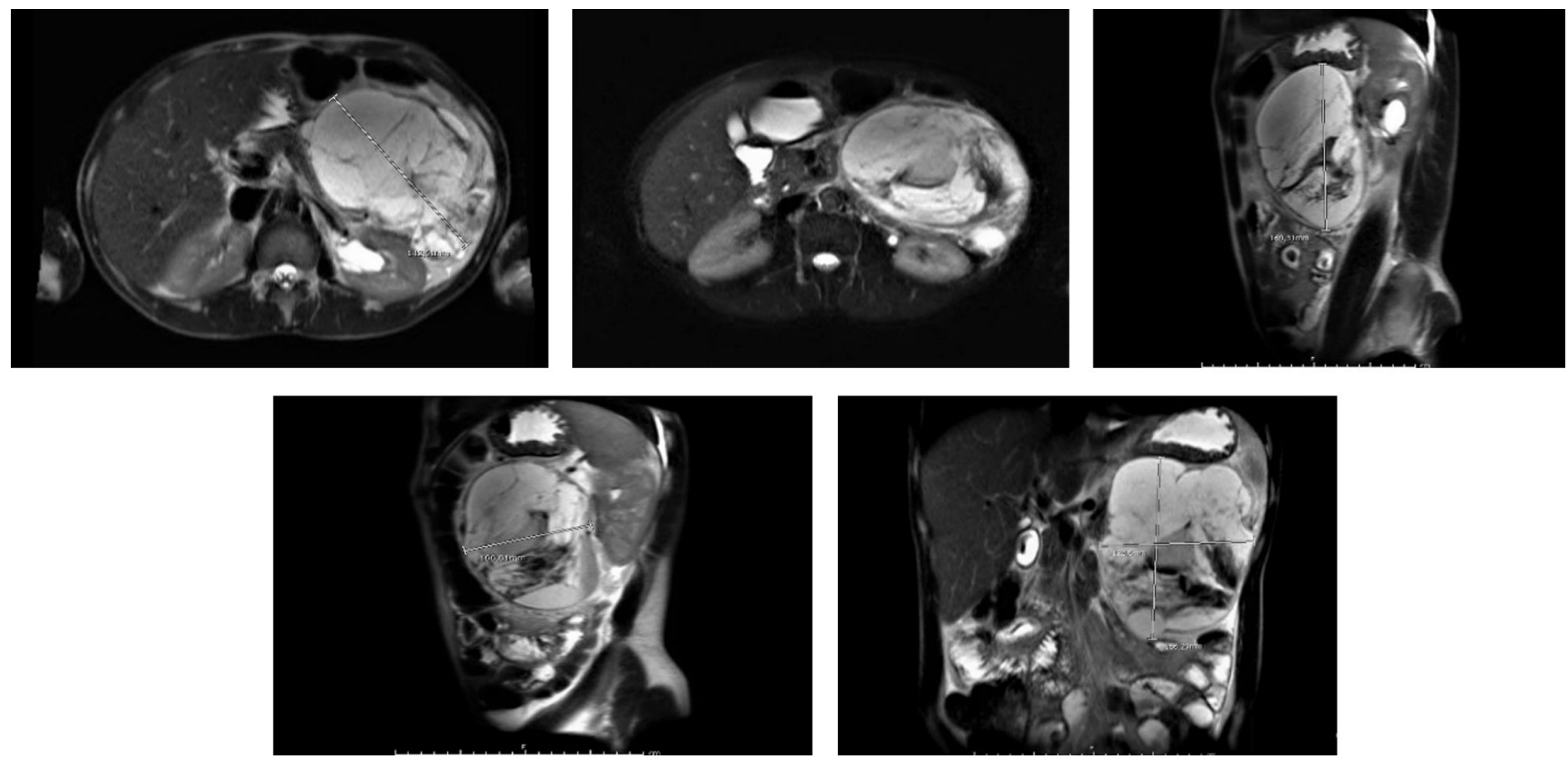

Figura 2. Estudio de resonancia magnética de abdomen.

nidos de aproximadamente 110 × $90 \mathrm{~mm}$ en el espacio pancreatoduodenal con desplazamiento del riñón izquierdo y un adecuado plano de clivaje con el páncreas en la región de la cabeza, cuerpo y cola sin lesiones focales; el resto de estudio no mostró ninguna alteración.

Con los hallazgos anteriores se llegó a la conclusión diagnóstica de quiste mesentérico, por lo cual se programó al paciente para procedimiento quirúrgico. Se realizó una laparotomía exploratoria en la que se encontró un tumor en el mesenterio del colon transverso hacia el ángulo esplénico de 150 × $200 \mathrm{~mm}$ de aspecto quístico sólido (Figura 3 ) firmemente adherido al bazo, cola de páncreas y colon, por lo que se realizó la resección del quiste mesentérico, esplenectomía, pancreatectomía distal y colectomía izquierda parcial con anastomosis laterolateral de colon. Por una pérdida sanguínea de $1200 \mathrm{~mL}$ aproximadamente e inestabilidad hemodinámica, el paciente requirió la transfusión de hemoderivados intraoperatorios e infusión de vasopresores. Su evolución posquirúrgica fue adecuada y se dio egreso a los 8 días del procedimiento quirúrgico. Se realizó control posquirúrgico ambulatorio a los 2 meses, el cual fue normal. 


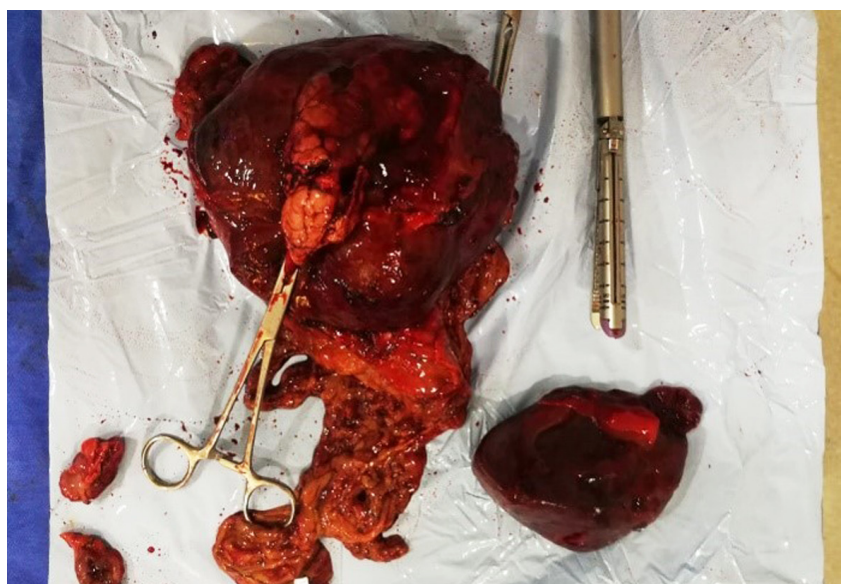

Figura 3. Masa obtenida en la resección quirúrgica.

El reporte final de patología reportó:

- Descripción macroscópica: resección en bloque, que consta del intestino grueso, con medidas de $19 \times 2,5 \mathrm{~cm}$; hacia el mesenterio transverso del colon se identificó una lesión irregular de color pardo de $15 \times 11 \mathrm{~cm}$, con superficie externa lisa y al corte se encontró cavitada y multiloculada, con un cambio hemorrágico extenso y salida de líquido hemático, la cual se encontró firmemente adherida al cuerpo del páncreas y midió $9 \times 4 \mathrm{~cm}$. El bazo pesaba 150 g y midió 12 x 9 x $2,5 \mathrm{~cm}$; a los cortes se encontró sin lesiones macroscópicas aparentes.

- Descripción microscópica: pared de colon sin evidencia de lesión. En los cortes de la pared intestinal, tumor y área tumoral en relación con el páncreas se observó una lesión caracterizada por la presencia de tabiques fibrosos sin recubrimiento epitelial con infiltrado mixto constituido por polimorfonucleares y plasmocitos asociados con una extensa hemorragia reciente; la lesión se encontró adherida al mesenterio de colon y al tejido adiposo peripancreático sin compromiso intersticial de los mismos. En el corte del bazo se identificó tejido esplénico con arquitectura conservada y una adecuada relación de la sustancia roja y blanca, sin lesiones histopatológicas.

- Diagnóstico:

- Resección en bloque de lesión mesentérica: quiste mesentérico con tamaño de $15 \times 11 \mathrm{~cm}$, colon y páncreas sin evidencia de lesión.

- Bazo sin alteraciones histopatológicas.

\section{DISCUSIÓN}

Los quistes mesentéricos tienen una baja incidencia mundial, de aproximadamente 1 de cada 100000 adultos que buscan atención médica, $y$ afectan principalmente a las personas en su segunda a tercera décadas de vida con una proporción de 2: 1 de mujer a hombre $(8,9)$. Estas lesiones pueden afectar cualquier parte del mesenterio y representan el $7 \%$ de todos los quistes abdominales (10). Estas lesiones exhiben características principalmente benignas, pero hasta el $3 \%$ de estas lesiones puede ser maligna. Se desconoce la causa del quiste mesentérico; sin embargo, la teoría más aceptada propuesta por Gross sugiere que es una proliferación benigna de tejido linfático mesentérico asociado con una falta de comunicación de los sistemas linfático y venoso, que puede ser idiopática o secundaria a procedimientos quirúrgicos previos, infección, enfermedad neoplásica, endometriosis o enfermedad inflamatoria pélvica (11).

El diagnóstico de quistes mesentéricos es difícil de realizar, ya que comúnmente imita otras afecciones como la enfermedad inflamatoria pélvica, el pseudoquiste pancreático y los aneurismas aórticos (12). La sospecha diagnóstica se puede tener en 3 categorías diferentes: síntomas abdominales inespecíficos, hallazgos radiológicos incidentales o abdomen agudo (13). Algunas complicaciones reportadas incluyen obstrucción intestinal, sangrado, ruptura, torsión intestinal o vólvulo (14).

Los estudios de imagen son importantes para el diagnóstico preoperatorio. Las diferentes modalidades de imagen tienen diferentes beneficios clínicos, pero las imágenes de TAC y resonancia magnética son más importantes para determinar una ubicación precisa, así como su relación con otras estructuras vecinas (14). Se establece un diagnóstico definitivo mediante la escisión quirúrgica y el examen histopatológico de la masa. Todas estas consideraciones fueron tomadas en cuenta en la gestión de nuestro caso.

La resección quirúrgica de la masa quística es la base del tratamiento y ha demostrado evitar la recurrencia y la transformación maligna $(15,16)$. La vía laparoscópica es la más segura y reduce el dolor posoperatorio, acorta la duración de la estancia hospitalaria y ha demostrado mejores tasas de recuperación. En nuestro caso, la laparoscopia no fue posible debido al tamaño de la lesión, pero se utilizó analgesia peridural y se tomaron medidas de deambulación temprana para la prevención de complicaciones. La aspiración simple no se recomienda debido a las mayores tasas de recurrencia e infección.

\section{CONCLUSIONES}

El quiste mesentérico es una patología rara y en su mayoría benigna, con presentación clínica inespecífica y retraso en el diagnóstico. Su proceso diagnóstico y caracterización se basan en estudios radiológicos, desde la ecografía hasta la resonancia magnética. El tratamiento de elección es quirúrgico, de este modo se evitan las recurrencias. En el caso clínico presentado con dolor abdominal luego de no tener mejoría, se realizaron estudios imagenológicos que evidenciaron una masa retroperitoneal con resección mediante el 
abordaje quirúrgico tipo laparotomía, cuya evolución posquirúrgica no tuvo complicaciones asociadas con el procedimiento ni con el reporte de histopatológico descrito.

\section{Agradecimientos}

Se agradece a todos los coautores por trabajar en este proyecto aportando su conocimiento y colaboración destinada en favor de la investigación; a la revista, por su respaldo, lo que ha permitido poder llevar a publicar este documento satisfactoriamente; y, finalmente, al Dr. Carlos Mauricio Martínez Montalvo, por su gran colaboración y apoyo en la presente investigación y en la vida.

\section{Conflictos de interés}

Los autores declaran que no presentan ningún conflicto de interés.

\section{Fuentes de financiación}

La financiación del presente se realizó con los aportes de los autores.

\section{Responsabilidades éticas}

Protección de personas y animales: los autores declaran que para esta investigación no se han realizado experimentos en seres humanos ni en animales.

Confidencialidad de los datos: los autores declaran que han seguido los protocolos de su centro de trabajo sobre la publicación de datos de pacientes.

Derecho a la privacidad y consentimiento informado: los autores han obtenido el consentimiento informado de los pacientes o sujetos referidos en el artículo. Este documento obra en poder del autor de correspondencia.

\section{REFERENCIAS}

1. Paramythiotis D, Bangeas P, Karakatsanis A, Iliadis A, Karayannopoulou G, Michalopoulos A. Ideal treatment strategy for chylous mesenteric cyst: a case report. J Med Case Rep. 2018;12(1):317. https://doi.org/10.1186/s13256-018-1716-x

2. de Perrot M, Bründler M, Tötsch M, Mentha G, Morel P. Mesenteric cysts. Toward less confusion? Dig Surg. 2000; 17(4):323-8 https://doi.org/10.1159/000018872

3. Kurtz RJ, Heimann TM, Holt J, Beck AR. Mesenteric and retroperitoneal cysts. Ann Surg. 1986;203(1):109-12. https://doi.org/10.1097/00000658-198601000-00017

4. Spolianski G, Kopelman D, Kimmel B, Hatoum OA. Laparoscopic exploration and treatment for a mesenteric cyst lymphangioma in adults. ANZ J Surg. 2019;89(10):1334-1336. https://doi.org/10.1111/ans.14711

5. Mullaney TG, D'Souza B. Mesenteric cyst: an uncommon cause of acute abdomen. ANZ J Surg. 2019;89(3):E98E99. https://doi.org/10.1111/ans.14067

6. Chou YH, Tiu CM, Lui WY, Chang T. Mesenteric and omental cysts: an ultrasonographic and clinical study of 15 patients. Gastrointest Radiol. 1991;16(4):311-4. https://doi.org/10.1007/BF01887376

7. Tan JJ, Tan KK, Chew SP. Mesenteric cysts: an institution experience over 14 years and review of literature. World J Surg. 2009;33(9):1961-5. https://doi.org/10.1007/s00268-009-0133-0

8. Ünlüer EE, Ünlüer S, Şahı N Y, Kamer KE, Karagöz A, Tan GC. An uncommon cause of abdominal pain: Mesenteric cyst. Interv Med Appl Sci. 2016;8(1):23-25. https://doi.org/10.1556/1646.8.2016.1.4

9. Sardi A, Parikh KJ, Singer JA, Minken SL. Mesenteric cysts. Am Surg. 1987;53(1):58-60.

10. Oh C, Danese CA, Dreiling DA. Chylous cysts of mesentery. Arch Surg. 1967;94(6):790-3. https://doi.org/10.1001/archsurg.1967.01330120044009

11. Leung BC, Sankey R, Fronza M, Maatouk M. Conservative approach to the acute management of a large mesenteric cyst. World J Clin Cases. 2017;5(9):360-363. https://doi.org/10.12998/wjcc.v5.19.360

12. Lee DL, Madhuvrata P, Reed MW, Balasubramanian SP. Chylous mesenteric cyst: A diagnostic dilemma. Asian J Surg. 2016;39(3):182-6. https://doi.org/10.1016/j.asjsur.2013.04.009

13. Liew SC, Glenn DC, Storey DW. Mesenteric cyst. Aust N Z J Surg. 1994;64(11):741-4. https://doi.org/10.1111/j.1445-2197.1994.tb04530.x

14. Yoshimitsu M, Emi M, Miguchi M, Ota H, Hakoda K, Omori I, et al. Single-incision laparoscopic excision of a chylous mesenteric cyst: A case report. Int J Surg Case Rep. 2016;29:254-257. https://doi.org/10.1016/j.ijscr.2016.11.029

15. Pithawa AK, Bansal AS, Kochar SP. "Mesenteric cyst: A rare intra-abdominal tumour”. Med J Armed Forces India. 2014;70(1):79-82. https://doi.org/10.1016/j.mjafi.2012.06.010

16. Dinçer M, Değer KC, Senger AS, Uzun O, Polat E, Duman $\mathrm{M}$, et al. Laparoscopic treatment of a mesenteric cyst. Prz Gastroenterol. 2016;11(2):143-4. https://doi.org/10.5114/pg.2015.55187 